\section{New Determination of the Magnetic Moment of the Proton in Terms of the Nuclear Magneton}

THe ratio of $f_{s}$, the spin precession frequency of the proton, to $f_{c}$, the cyclotron frequency, in the same magnetic field is equal to the ratio of the magnetic moment of the proton $\mu_{p}$ to the nuclear magneton ${ }^{1} \mu_{n}$.

$$
f_{d} / f_{c}=\mu_{p} / \mu_{n}
$$

The ratio is used at present in combination with other experimentally moasured constants to derive the best values for the atomic constants ${ }^{2}$. The uncertainties of current estimates of the Faraday constant, ratio of proton to electron mass, electronic charge, nuclidic mass unit and many other important constants are effectively determined by the uncertainty of $\mu_{p} / \mu_{n}$.

Our measurements were made at 0.47 tesla (4.7 k gauss). The spin precession froquency was moasured with an amplitude bridge ${ }^{3}$ and the cyclotron frequency using the omegatron method4. The omega tron was of the quadrupole type ${ }^{5}$ and had split plates to improve the uniformity of the radio frequency field. Ratios of the masses of ions are known with great procision and may be regarded as auxiliary constants ${ }^{2}$. The cyclotron frequencies of hydrogen ions $\left(\mathrm{H}_{2}^{+}\right)$, douterated hydrogen ion $\left(\mathrm{HD}^{+}\right)$and deuterium ions $\left(\mathrm{D}_{2}^{+}\right)$were measured, and this enabled the shifts in the cyclotron frequencies caused by electrostatic fields to bo eliminated.

The results of forty-three sets of three ion resonances, with standard deviations (including both systematic and random errors), are

$$
\begin{aligned}
& \mu_{p} / \mu_{n}=2.79274 \pm 0.00005 \text { without and } \\
& \mu_{p} / \mu_{n}=2.79282 \pm 0.00005 \text { (19 p.p.m.) with }
\end{aligned}
$$

the correction for the diamagnetism of the water sample ${ }^{6,7}$, respectively. This lies within $\mathbf{l} \cdot \mathbf{l}$ standard deviations of the present best value and midway between the values which were included ${ }^{4,8,0}$ in the 1965 evaluation and those which were rejected ${ }^{10}$ or too late ${ }^{11}$ to include. It is hoped to increase the precision of the method by a factor of ten by working at higher magnetic fields.

This work forms part of the research programme of the National Physical Laboratory.

\section{B. W. Petrey \\ K. MorRIS}

Quantum Metrology Division,

National Physical Laboratory,

Teddington,

Middlesex.

\section{Received January 5, 1967.}

${ }^{1}$ Alvarez, L. W., and Bloch, F., Phys, Rev., 57, 111 (1940).

"Cohen, W. R., and DuMond, J. W. M., Kev. Mod. Phyk., 37, 537 (1965).

${ }^{3}$ Thomas, H. A., and Huntoon, R. D., Rev. Sci. Instrum., 20, 516 (1949).

4 Sommer, H., Thomas, H. A., and Hipple, J. A., Phys. Rev., 82, 697-702 (1951).

s Petley, B. W., and Morris, K., J. Sci. Instrum., 42, 492 (1965).

- Ramsey, N. F., Phys, Rev., 78, 699 (1950).

'Myint, 'T.' Klepner, D., Ramsey, N. F., and Robinson, H. G., Phys, Rev. (1)

Rull. Amer. Phys. Soc., 1, 220 (1956)

- Sanders, J. H., and Turberfield, K. C., Proc. Roy. Soc, A, 272, 79 (1963).

${ }^{10}$ Boyne, H. S., and Franken, P. A., Phys. Rev.,123, 242 (1961).

"Manyrin, B. A., and Frantsuzov, A., J. Expt. Theoret. Phys. (U.S.S.R.) 48, 416 (1965) (English translation: Soviet Phys..JET $P, 21,274$ (1965))

\section{Air Flow with Arc Present}

THE bohaviour of a flowing gas when disturbed by the presence of a high current arc has always been of great interest to circuit-breaker engineers and physicists.

The intense light of the are under investigation is generally so much greater than that of the conventional background light source of the interferometer or Schlicron system used that it is extremely difficult, and in most cases quite impossible, to photograph the gas movement. This difficulty has been overcome by using a laser in place of the conventional light source of the interferometer system. 